\title{
Diffraction of optical beams by a half-plane
}

\author{
S.Anokhov, A.Khizhnyak, R.Lymarenko \\ International Center "Institute of Applied Optics», National Academy of Sciences of Ukraine, 254053, Kyiv, Ukraine \\ phone: 380(44)2122158,fax380(44)2124812,e-mail:khizh@writeme.com
}

\begin{abstract}
Rigorous solution of the optical beam diffraction problem on half plane is represented. Solution is described to representation of edge dislocation wave (EDW) that was introduced for describing the diffraction plane wave on half plane. It is shown that in problem of diffraction EDW plays the role of eigenmode as plane wave in free space. Due to this the solving of mentioned problem reduces to calculation of convolution of angle spectrum of source beam and EDW.
\end{abstract}

Keywords: diffraction theory, Gaussian beam.

Paper received 19.11.99; revised manuscript received 15.12.99; accepted for publication 04.01.00.

\section{Introduction}

Diffraction of plane electromagnetic wave on half plane and apertures will be easy calculated on base of the Kirchhoff theory [1]. But in the case of non-planar waves and finite beams results of Kirchhoff's description are not rigorous [2]. Because there is no universal method of soving problems of such sort, the interest for investigation diffraction of real beams on different apertures [514] is understandable.

The simplest problem of beam diffraction is the case of diffraction beam on half-plane. First quantitative investigation of Gaussian beam diffraction on half plane was made three decades before [15], but traditional point of view did not give authors any possibility to obtain new results. More rigorous solution was realized on the base of the wave equation [16] but due to mathematical difficulties authors stopped on equations in paraxial approximation.

In our previous paper [17] introduced was the new representation of diffracted field for plane wave and halfplane as screen. It was shown that this field might be split on two partial waves: the source plane wave with half amplitude and new wave that was denominated as edge dislocation wave (EDW). This wave has edge dislocation in plane that passes through the edge of half-plane and coincides with the geometric shadow boundary.

Problem of optical beam diffraction is a linear problem and can be solved by decomposition method by eigenmodes. In the case of diffraction on half plane, the role of an eigenmode is played by EDW. Really, the dif- fracted field of plane wave is a sum of two components [17]. In free space arbitrary beam can be represented as a sum of plane waves. When this beam impinges on halfplane, it is possible to solve diffraction problem for each plane wave on this aperture independently one from another. A final result will be the sum of diffracted fields of each plane wave. This means that we can introduce the field of the diffracted beam as the sum of field source beam with half amplitude and other beam which is the superposition of EDW that represents the diffracted field of each plane wave compounded the source beam.

\section{Theoretical consideration}

\subsection{General approach}

Without loss of generalities [1] and only for simplicity let's investigate 2-dimensional problem (Fig. 1). As it was shown in our paper [17], the diffracted field of a zpolarized plane wave with amplitude $\mathrm{A}_{0}$ is described after the screen by the following expression:

$$
\begin{aligned}
& E_{z}(x, y)=\frac{A_{0}}{2} \exp \left(i\left(k_{x} x+k_{y} y\right)+\right. \\
& +A_{0} \frac{1-i}{\sqrt{2 \pi}} \times f(U) \times \exp \left(i\left(k_{x} x+k_{y} y\right),\right.
\end{aligned}
$$

where 


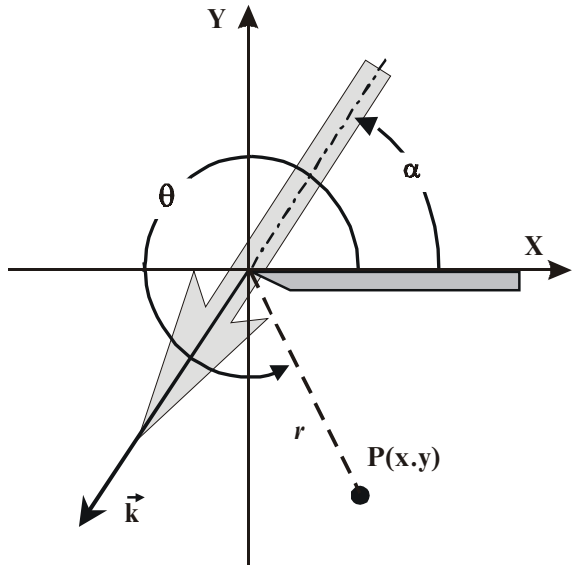

Fig. 1. The scheme of beam diffraction on a half-plane. $k$ is a wave vector of partial plane wave composing an incident beam.

$U= \pm \sqrt{k \sqrt{x^{2}+y^{2}}+k_{x} x+\sqrt{k^{2}-k_{x}^{2}} y}$

and sign «+» is used for $x<0$, and vice versa;

$f(U)=\int_{0}^{U} \exp \left(i \mu^{2}\right) d \mu$,

is the Fresnel integral, $k=2 \pi / \lambda ; \lambda$ is a wavelength; $k_{x}=$ $=k \sin \left(\alpha_{0}\right) ; k_{y}=k \cos \left(\alpha_{0}\right) ; \alpha_{0}$ is an angle of the source wave propagation (angle between wavevector and $X$-axes Fig. 1).

The first summand in Exp.(1) describes a plane wave that coincides with input plane wave but has a half of its amplitude. The second summand describes EDW that is shown in Fig. 2. Exp.(1) can be interpreted as follows. Behind the screen, the source plane wave is disintegrated on two parts: the first is the same plane wave but of half amplitude. The second part is an infinite wave which has edge dislocation, coincides with the boundary of a geometrical shadow, i.e., screen contributes phase shift on $p$ into parts of plane wave that is blocked by half plane.

Arbitrary z-polarized 2D beam can be represented by its spatial spectrum:

$$
\begin{aligned}
& B_{z}(x, y) \exp (-\vec{k} \vec{r})= \\
& =\int_{-\infty}^{\infty} F_{B}\left(k_{x}\right) \times \exp \left(-i k_{x} x-i \sqrt{k^{2}-k_{x}^{2}} y\right) d k_{x} .
\end{aligned}
$$

After the half-plane (Fig. 1) the field of each plane wave is transformed and described by Exp.(1). Substitution of Exp.(1) inside Exp.(4) represents the field after the screen as the sum of two components:
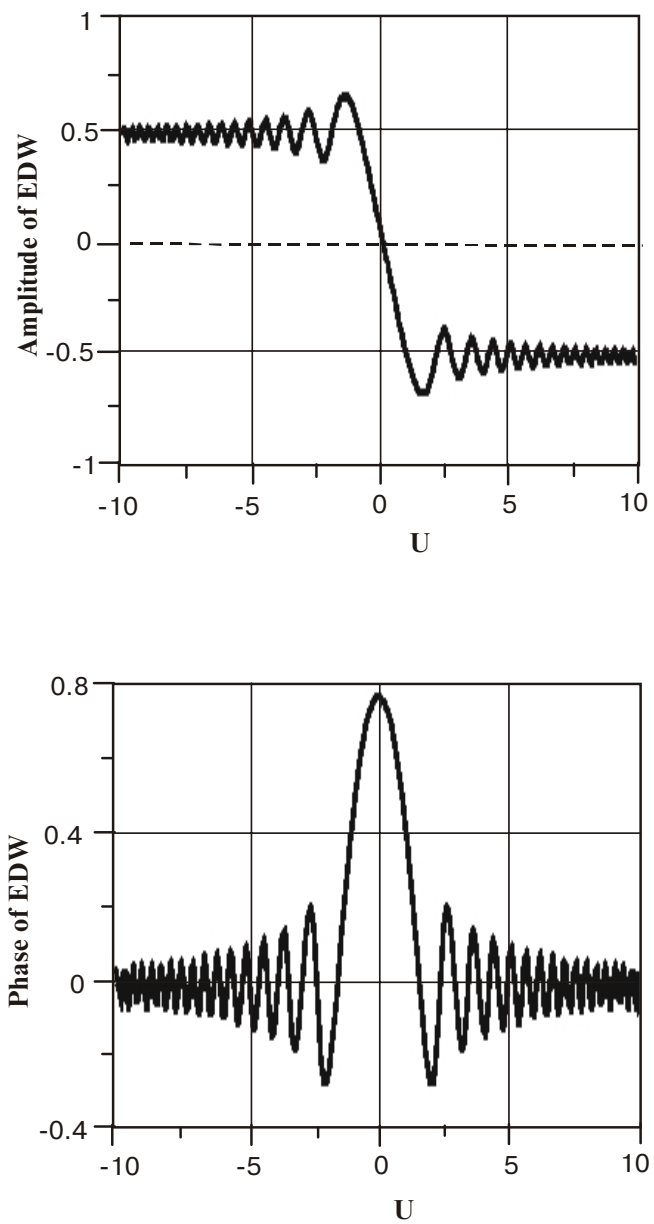

Fig. 2. Structure of EDW, its amplitude and phase.

$$
D_{z}(x, y)=\frac{1}{2} B_{z}(x, y) \times \exp (-i \vec{k} \vec{r})+
$$$$
+E_{E D B}(x, y)
$$

The first component is the source beam with half amplitude, and the second is the beam which is superposition of EDW and lets to denominate it as an edge dislocated beam (EDB) because it has an edge dislocation exactly near the screen:

$$
E_{E D B}(x, y)=\frac{1-i}{\sqrt{2 \pi}} \times
$$

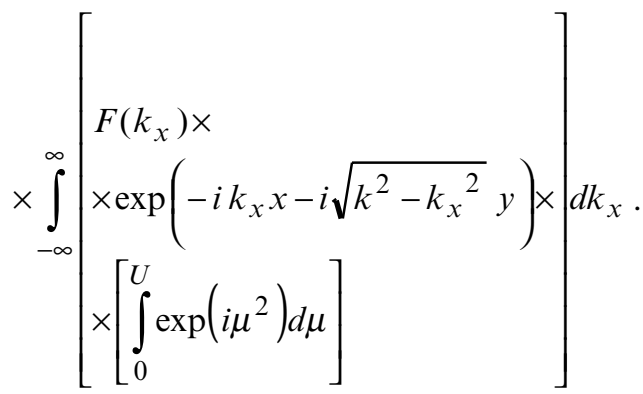


Exp.(6) may be transformed owing to integration by parts and taken into account finitness of the beam and its spatial spectrum:

$$
\begin{aligned}
& E_{E D B}(x, y)=-\frac{(1+i) \sqrt{2 \pi}}{4} \times \\
& \times \exp \left(-i k \sqrt{x^{2}+y^{2}}\right) \times \\
& \times \int_{-\infty}^{\infty} \frac{d}{d U}\left[\frac{F(U)}{\varphi(U)}\right] \times \Phi(U) d U,
\end{aligned}
$$

where $\varphi(U)=\frac{d U}{d k_{x}}$

$$
\Phi(U)=\left\{\frac{1-i}{\pi}\left[\int_{0}^{U} \exp \left(i \mu^{2}\right) d \mu\right]^{2}-\frac{1}{4}\right\}
$$

Function $\Phi(U)$ is localized as it is shown on Fig. 3, and its halfwidth is $\Delta \Phi(U)=4$. Derivative in the expression under integral can be written in the following form:

$$
\frac{d}{d U}\left[\frac{F(U)}{\varphi(U)}\right]=\left\{\frac{\frac{d F\left(k_{x}\right)}{d k_{x}}}{\left(\frac{d U\left(k_{x}\right)}{d k_{x}}\right)^{2}}-F\left(k_{x}\right) \frac{\frac{d^{2} U\left(k_{x}\right)}{d k_{x}^{2}}}{\left(\frac{d U\left(k_{x}\right)}{d k_{x}}\right)^{3}}\right\}
$$

after differentiation it is need to substitute $k_{x}$ :

$$
k_{x}=\frac{x\left(k r-U^{2}\right) \pm y U \sqrt{2 k r-U^{2}}}{r^{2}},
$$

where $r^{2}=x^{2}+y^{2}$.

\subsection{Paraxial approximation}

Practically, in most cases, paraxial beams are used, i.e. that satisfying conditions: $k_{x}<<k_{y}, x<<$. In this case variable $U$ can be simplified:

$$
\bar{U}=\sqrt{\frac{k y}{2}}(\Delta-\delta),
$$

where $\delta=k_{x} / k, \Delta=x / y-$ angle between $\mathrm{Y}$-axes and direction in point $\mathrm{P}$ (Fig. 1), and simplified Exp.(10):

$$
\frac{d}{d \bar{U}}\left[\frac{F(\bar{U})}{\varphi(\bar{U})}\right]=\sqrt{\frac{2 k}{y}} \times \frac{d F(\bar{U})}{d \bar{U}}
$$

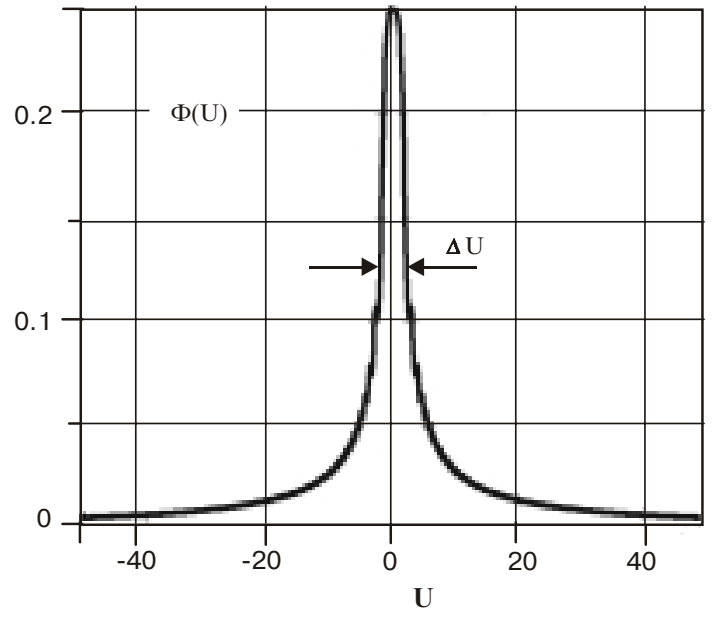

Fig. 3. Amplitude of the factor in square brackets of Eq.(10). Halfwidth is equal to 4 .

and Exp.(7) is transformed, too:

$$
\begin{aligned}
& E_{E D B}(x, y)=-\sqrt{2 \pi} \times \frac{(1+i)}{2} \times \sqrt{\frac{2 k}{y}} \times \\
& \times \exp \left(-i k \sqrt{x^{2}+y^{2}}\right) \times \\
& \times \int_{-\infty}^{\infty} \frac{d F(\bar{U})}{d \bar{U}} \times \Phi(\bar{U}) d \bar{U}
\end{aligned}
$$

\subsection{Far field}

For a beam having width $d$ on the screen, the scale in a far field will be not less than $k_{x} / k>\lambda / d$ and in units $\bar{U}_{d}$ $\bar{U}_{d}>\sqrt{\pi / N}$, where $N=d^{2} /(\lambda y)$ is the Fresnel parameter. So for the far field, when $N$ goes to zero, the function $\Phi(\bar{U})$ is a $\delta$ - function and solution (14) will be simplified:

$$
\begin{aligned}
& E_{E D B}(x, y)=-\sqrt{2 \pi} \times \frac{(1+i)}{2} \times \sqrt{\frac{2 k}{y}} \times \\
& \times \exp \left(-i k \sqrt{x^{2}+y^{2}}\right) \\
& \times\left.\frac{d F(\bar{U})}{d \bar{U}}\right|_{U=0} \times D
\end{aligned}
$$

where constant $D$ is

$$
\begin{aligned}
& D=\int_{-\infty}^{\infty}\left[\left(\int_{0}^{U} \exp \left(i \mu^{2}\right) d \mu\right)^{2}-\frac{1}{4}\right] d \bar{U} \cong \\
& \cong 0.43023 \times e^{-i 2.3345}
\end{aligned}
$$


Exp.(15) shows quite unexpected, but beautiful resultEDB in the far field of an arbitrary beam is described by derivative of its spatial spectrum. So the far field of arbitrary beam diffracted on half-plane is an algebraic sum of the beam's spatial spectrum and derivative of this spectrum.

\section{Diffraction of Gaussian beam}

For demonstration possibilities of our solution, let us investigate the case of the often-used Gaussian beam. Typically, the lowest transverse laser mode has a structure like Gaussian beam, which can be written for a waist situated on the screen in the following form:

$$
E(x, y)=\exp \left(-\frac{\left(x-x_{0}\right)^{2}}{a^{2}}\right) \times \exp (i k y),
$$

where $a$ is a beam diameter, $x_{0}$ is a value of the beam maximum shifting from the edge of the screen. Fourier spectrum of this beam is:

$$
F\left(k_{x}\right)=a \times \exp \left(-\frac{k_{x}^{2} a^{2}}{4}-i k_{x} x_{0}\right) .
$$

\subsection{Paraxial approximation}

In this approximation Exp.(18) can be rewritten in the form:

$$
\begin{aligned}
& F(\bar{U})=a \times \exp \left(-\frac{x_{0}{ }^{2}}{a^{2}}\right) \times \\
& \times \exp \left[-\left(\sqrt{\pi N} \times \bar{U}-\frac{k a}{2} \Delta+i \frac{x_{0}}{a}\right)^{2}\right],
\end{aligned}
$$

where $N=a^{2} /(\lambda y)$ is the Fresnel number.

Substitution of Exp.(17) in (14) gives the solution for EDB in paraxial approximation:

$$
\begin{aligned}
& E_{E D B}(x, y)=2 \sqrt{2 \pi} \times \frac{(1+i)}{2} \times \frac{k a^{2}}{y} \times \\
& \times \exp \left(-i k \sqrt{x^{2}+y^{2}}\right) \times \exp \left(-\frac{x_{0}^{2}}{a^{2}}\right) \times \\
& \times \int_{-\infty}^{\infty}\left[\left(\sqrt{\pi N} \times \bar{U}-\frac{k a}{2} \Delta+i \frac{x_{0}}{a}\right) \times\right. \\
& \left.\times \exp -\left(\sqrt{\pi N} \times \bar{U}-\frac{k a}{2} \Delta+i \frac{x_{0}}{a}\right)^{2}\right] \times \Phi(\bar{U}) d \bar{U}
\end{aligned}
$$

It is seen that only one parameter, the Fresnel number, determines the behavior of EDB, but its value has not to be very high in paraxial approximation.

\subsection{Far field}

The simplest type of solution is got from Exp.(20) for the EDB far field distribution when $N$ goes to zero:

$$
\begin{aligned}
& E_{E D B}(x, y)=-\sqrt{2 \pi} \times(1+i) \times \frac{k a^{2}}{y} \times \\
& \times \exp \left(-i k \sqrt{x^{2}+y^{2}}\right) \times D \times \\
& \times\left(\frac{k a}{2} \Delta-i \frac{x_{0}}{a}\right) \times \\
& \times \exp \left[-\left(\frac{k a}{2} \Delta\right)^{2}-i k x_{0} \Delta\right]
\end{aligned}
$$

and EDB intensity distribution in far field normalized on its maximum describes the following expression:

$$
\begin{aligned}
& J(\Delta)=\frac{I_{E D B}^{(f f)}}{I_{E D B}^{(\max )}}= \\
& \int 2 \times\left[\left(\frac{k a}{2} \Delta\right)^{2}+\left(\frac{x_{0}}{a}\right)^{2}\right] \times \\
& =\left\{\times \exp \left[1-2 \times\left(\frac{x_{0}}{a}\right)^{2}-2 \times\left(\frac{k a}{2} \Delta\right)^{2}\right], \quad x_{0} \leq a / \sqrt{2}\right. \\
& \left\{\left(\frac{a}{x_{0}}\right)^{2} \times\left[\left(\frac{k a}{2} \Delta\right)^{2}+\left(\frac{x_{0}}{a}\right)^{2}\right] \times\right. \\
& \times \exp \left[-2 \times\left(\frac{k a}{2} \Delta\right)^{2}\right], \quad x_{0}>a / \sqrt{2}
\end{aligned}
$$

It is clear that function $J(\Delta)$ is symmetrical function of its argument $\Delta$ and has zero for $\Delta=0$ only in the case $x_{0}=0$, i.e. when the beam axis crosses the edge of the screen. Only in this case there is the edge dislocation for $\Delta=0$ because both real and imagine parts of field are equal to zero. Fig. 4a demonstrates intensity distribution of EDB, and its phase front for different values of shifting $x_{0} / a$. The phase structure of EDB field $\Psi\left(\Delta, x_{0}\right)$ (Exp.21) is shown in Fig. 4b.

Fig. 4 shows that phase front has hop on $\pi$ for $x_{0}=0$ when there is edge dislocation and tilt for $x_{0} \neq 0$, moreover the sign of tilt depends on sign of $x_{0}$, i.e. direction of beam axis shift from edge of screen. 


\subsection{Intermediate distance}

If the Fresnel number is not very high, we can use paraxial approximation. In this area EDB is described by Exp.(20), which can easy calculate. Examples of intensity distribution and phase front are shown in Fig. 5. The figure shows EDB to be very closed to EDW (Fig.2), though EDB has an amplitude decreasing in transversal direction because the Gaussian beam amplitude decreases in this direction, too.

\section{Experimental results}

\subsection{Experimental setup}

Being encouraged by the new look on the wave composition of a diffraction field, we performed a direct experiment on isolation and detection of the EDB. The experimental scheme of Mach-Zehnder interferometer for observation and investigation of the forward-propagated EDW is shown in Fig. 6.

Single-mode linearly polarized $\mathrm{He}-\mathrm{Ne}$ laser radiation was extended by telescope. The beam was splitted
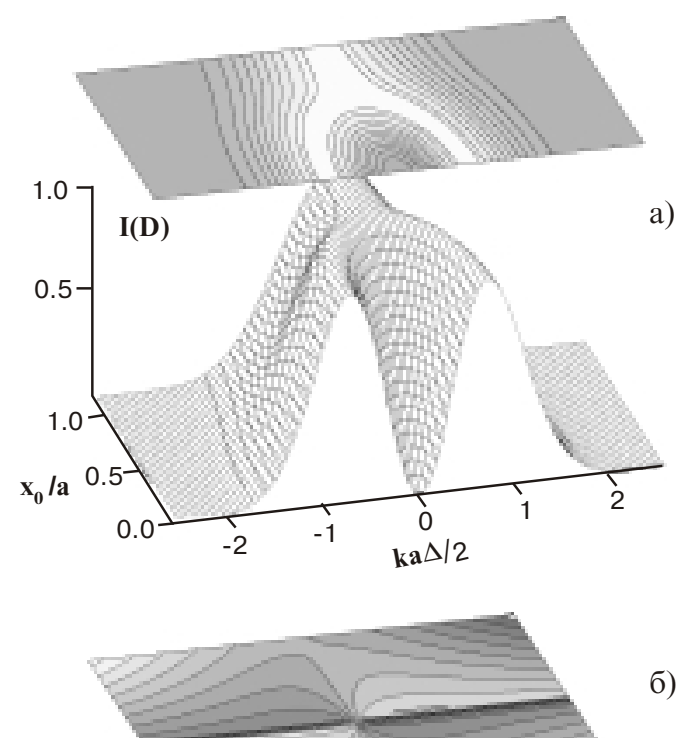

Fig. 4. The structure of intensity distribution of EDB and its phase front in the far field for different values of the shift, $x_{0} / a$.
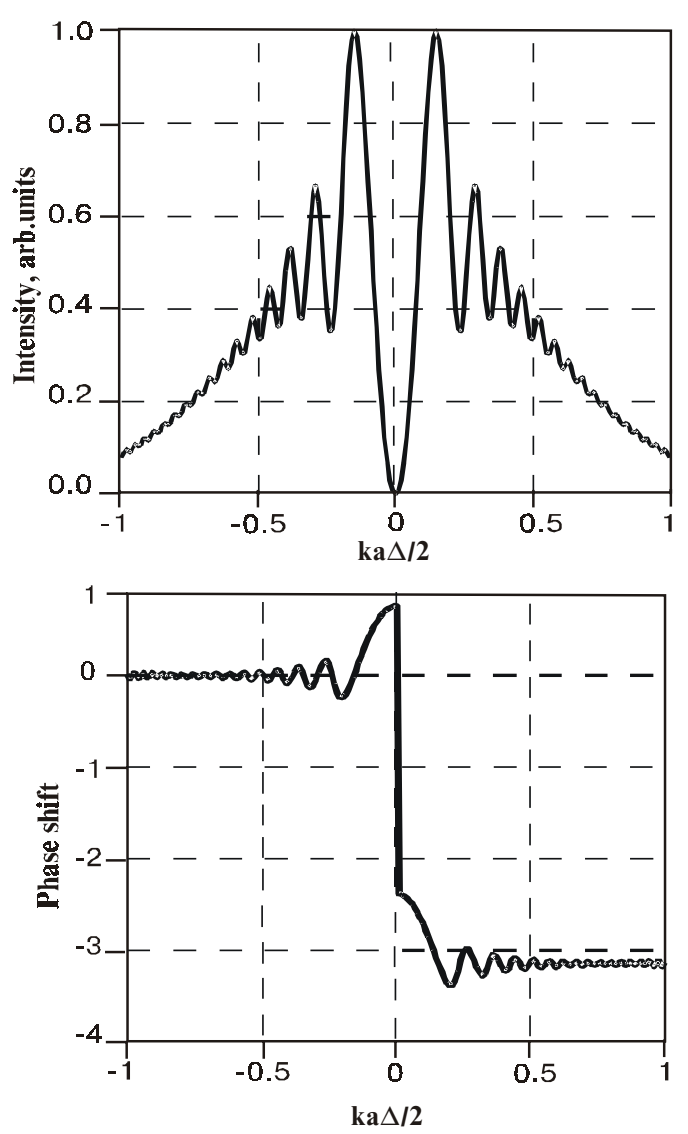

Fig. 5. Structure of EDB in near field $(C=100)$, its amplitude and phase.

into equal parts with the aid of beam-splitter cube with face dimensions $20 \times 20 \mathrm{~mm}$ and $50 \%$ division coefficient. The directly transmitted beam was attenuated to a necessary degree by a variable filter 4 and after reflection from the mirror 6 was directed to the observation plane 9 through the splitter 5 identical to the element 3 . In another arm a beam reflected from a piezodrived mirror 7 diffracted on an edge of a razor blade cutting off a half of its aperture. The role of piezomirror was to control the relative phase between the interfering waves at the observation plane. A CCD camera was placed there with the aperture $4.8 \times 3.2 \mathrm{~mm}^{2}$. The signal from CCD camera was directed to the computer. A lens with the large focal length was used for far field observation.

\subsection{Near field}

Intensity distribution of diffracted field looks like a field of diffracted plane wave (Fig. 7a), but, unlike plane wave, an average intensity decreases for displacement out of boundary of geometrical shadow. Distributions of light bands are the same as for plane wave diffraction, but average intensity decreases together with decreasing intensity of beam. Shifting the beam axis out of the screen edge leads to changes of light and dark values of diffracted beam. 


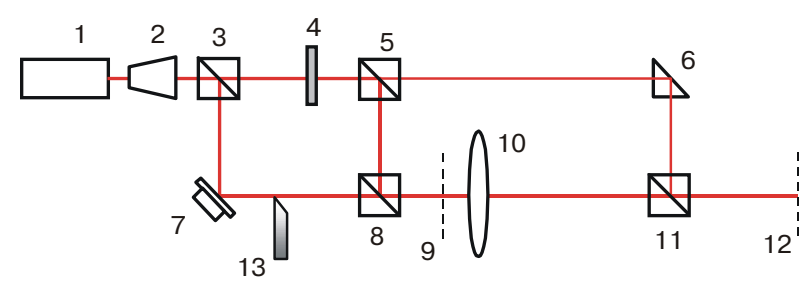

Fig. 6. Experimental set-up: 1 - He-Ne laser, 2 - telescopic beam expander (plane-wave former), 3,5,8,11 - beam splitters $(50 \%)$, 4 - variable density filter, 6 - mirror, 7 - mirror mounted on piezoshifter, $10-$ lens with focal distance $\mathrm{f}=1100 \mathrm{~mm}, 9,12-$ image plane with CCD camera, 13 - sharp-edge screen.

For selection of EDB, diffracted field interferes with reference beam a phase of which was shifted by $p$ due to piezomirror. Fig. 7b demonstrates intensity distribution of EDB on the distance of $25 \mathrm{~cm}$ from the screen provided the beam axes goes through the screen edge. As Fig. 7b shows, in this case, there is symmetrical intensity distribution and edge dislocation that coincides with a border of geometrical shadow. When beam axes is shifted from the screen edge, EDB becomes asymmetrical (Fig. 8), and edge dislocation moves on the beam periphery into the weak part of the beam.

The reason of the edge dislocation pulling is asymmetry of the field behind the screen. For sufficiently broad beam near the screen, its asymmetry is rather small, and edge dislocation can be born. But with growing distance from the screen balance of beam's left and right parts breaks, and edge dislocation is pulled on the beam periphery into the side of the weaker part of the beam. As a result, the wavefront gets the tilt, which is shown on an interference picture with additional reference beam (Fig. 7c). The observed interference fringes were shifted on a half of the period along the dislocation line, which corresponds to the $\mathrm{p}$-shift. Also, phase modulation around the dislocation line following from the theoretical predictions was revealed.

Fig. 9 demonstrates the transformation of the intensity of forward propagating EDB. The spatial evolution of EDB likes the EDW but not only scaling of the transverse amplitude distribution occurs.

\subsection{Far field}

Some important events happen behind the screen in the far field. As it was noted above, EDB far field is symmetrical for any value of the beam shifting through the screen edge, what is confirmed in the experiment. It was pointed out that the dislocation in EDB disappeared under inflexion or shifting of the incident beam center from screen edge. At the same time, the symmetry of the far field was not perverted whichever appreciable shifting the diffracted beam.

The Fig. 10 illustrates general behavior of the EDB far field structure when the screen edge goes through the beam axis. As shown above, while shading the beam is low, the far field of EDB presented a uniform structure without any singularities. With increase of the beam screening the ravine is formed, which reached zero level under matching the beam center and the screen edge. At further shifting the screen, the evolution of the EDW far field repeats considered phases backward.

Wave-front shape of the diffraction field was examined with the tilted reference quasi-plane wave (Fig. 11). The observed interference picture is unequivocally characterized the phase structure of the diffracted field. The phase difference between lobes of the far field distribution grades 0 into $\pi$. Specifically, the sign of the phase increment indicates the direction of screen movement over the beam center.

The totality of experimental results substantiated the theoretical conclusions and proved the behavior (especially symmetry) of the EDB far field (compare experimental distribution Fig.10 and 11 and calculated distribution Fig.4).

\section{Conclusions}

In summary, the new approach can be beneficial to the study of the diffraction phenomena, which based on the investigation the most informative part of diffracted fieldedge dislocation wave. In the case of the Gauss beam diffraction, the EDW transformed into beam with edge dislocation (EDB). Considerable achievements of new interpretation are a gracious union of numerical precise rigorous mathematical solution and possibility of deeper

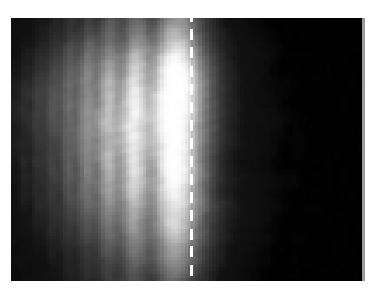

a)

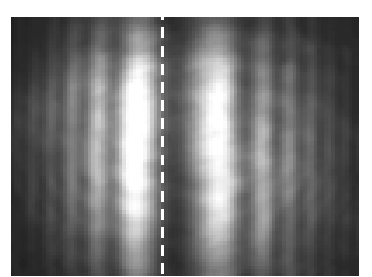

b)

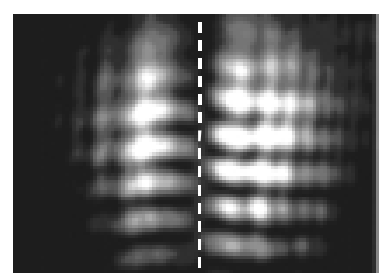

c)

Fig. 7. Experimental pictures (in gray scale) of intensity distribution behind the screen (a). Intensity distribution of the isolated EDB appearing when half-amplitude incident wave component is subtracted from the diffraction field (b). The pattern interference between EDB and tilted reference quasi-plane wave (c). Dashed line shows the position of edge dislocation. 


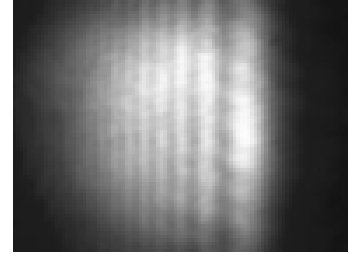

a)

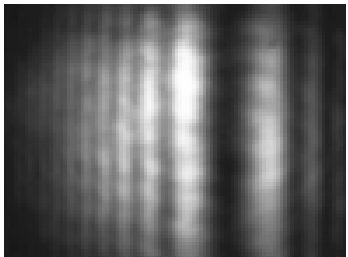

b)

Fig. 8. Intensity distribution of the diffracted field (a) and asymmetric EDB (b) when the screen edge is shifted from the beam center. The incident Gaussian beam is centered on the pictures.
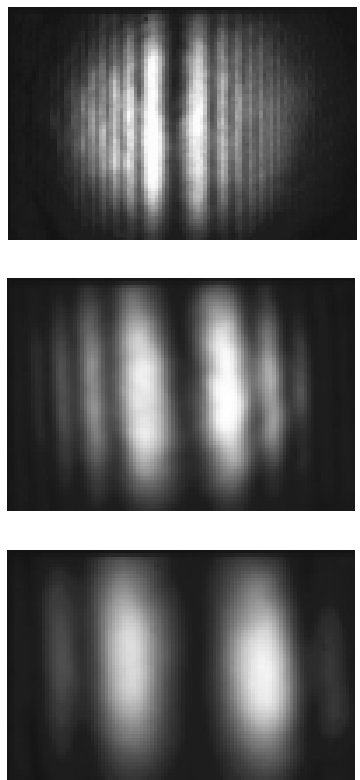

Fig. 9. Spatial evolution of EDB, distance from the edge screen is equal to $0.25,1$ and $2 \mathrm{~m}$.
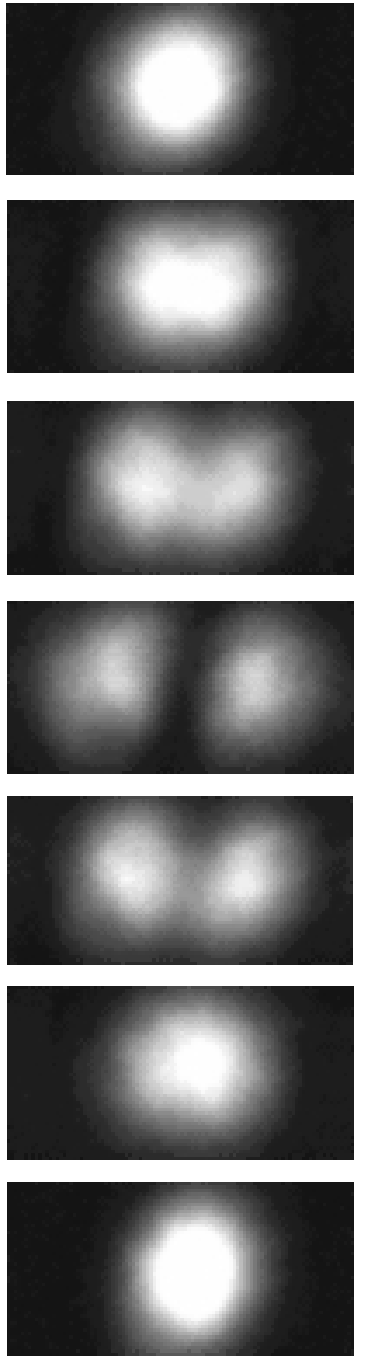

Fig. 10. The evolution of the far field of EDB when the screen edge goes through the beam axis.
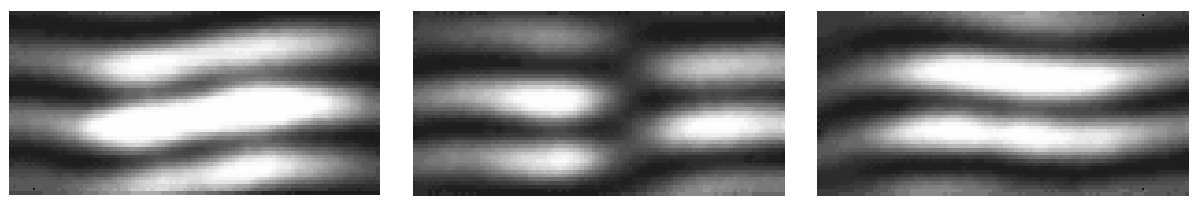

Fig. 11. Experimental pictures of intensity distribution interference between EDB far field and tilted reference plane wave (corresponding frames $3,4,5$ of fig. 9 , respectively).

circumstantiation of the wave process. This last particularity allows to reveal the new qualitative characteristic of this phenomena. This will not be able to succeed in the traditional ways.

Results of this paper brightly illustrate the possibility of new approach to describe some diffraction phenomena, particularly for far field distribution, when solu- tion can be presented as a simple algebraic sum of spatial spectrum and its derivative.

The pattern of formation of the diffracted beam far field is appreciably refined and the evolution of it's phase structure depends on position of the incident beam center over the edge of the screen. In particular, when the beam center is placed on the edge exactly, this wave possess 


\section{S.Anokhov, A.Khizhnyak, R.Lymarenko: Diffraction of optical beams by a half-plane}

infinitely-extended edge dislocation of their wave fronts (plane of the wave front break) located exactly on the border of geometrical shadow. A simple method of the edge visualization may be based on it and can be useful in practical applications.

\section{References}

1. M. Born, E. Wolf, Principles of optics, 6-th edition, Pergamon Press «Oxford» (1991).

2. Y. Li, Oscillations and discontinuity in the focal shift of Gaussian laser beams // Opt. Soc. Am., A3, 1761-1765 (1986)

3. A.N. Smirnov, S.A. Strokovskii // Optics and Spectroscopy, 76, N6, pp. 1019-1026 (1994).

4. S. Wang, On principles of difraction // Optik, 100, N3, 107108 (1995)

5. J.P. Campbell, L.G. DeShazer, Near fields of truncated Gaussian apertures // J. Opt. Soc. Am., 59, 1427-1429 (1969).

6. D.A. Holmes, J.E. Korka, P.V. Avizonis, Parametric Study of Apertured Focused Gaussian Beams // Appl. Opt., 11, 565-574 (1972).

7. K. Tanaka, M. Shibukawa, O. Kukumitsu, Difraction of a Wave Beam by an Aperture // IEEE Trans. Microwave Theory Tech., MTT-20, 749-754 (1972).

8. P. Belland, J.P.Crenn, Changes in the characteristics of a Gaussian beam weakly diffracted by a circular aperture // Appl. Opt., 21, 522 -527 (1982).
9. K. Tanaka, N. Saga, H. Mizokami, Field spread of a diffracted Gaussian beam through a circular aperture // Appl. Opt., 24, 1102-1106 (1985).

10. V.N. Mahajan, Uniform versus Gaussian beams: a comparison of the effects of digraction, obscuration and aberrations // J. Opt. Soc. Am., A3, 470-485 (1986).

11. Y. Li, Degeneracy in the Fraunhofer difraction of truncated Gaussian beams // J. Opt. Soc. Am., A4, 1237-1242 (1987).

12. S. Wang, E. Bernbeu, J. Alda, ABCD matrix for weakly apertured Gaussian beams in the far-feald // Appl. Opt., 30, 1584-1585 (1991).

13. R. Martinez-Herrero, P.M. Mejias, M. Arias, Parametric characterization of coherent, lowest-order Gaussian beams propagating through hard-edged apertures // Opt. Lett., 20, 124-126 (1995).

14. H.T. Yura, T.S. Rose, Gaussian beam transfer through hardaperture optics // Appl. Opt., 34, 6826-6828 (1995).

15. J.E. Pearson, T.C. McGill, S. Kurtin, A. Yariv, Diffraction of Gaussian laser beams by a semi-infinite plane // J. Opt. Soc. Am., 59, N11, 1440-1445 (1969).

16. P. Hillion, Diffraction of a Gaussian beam at a perfectly conducting half-screen // Opt., 26, N2, 57-64 (1995).

17. A.I. Khizhnyak, S.P. Anokhov, R.A. Lymarenko, M.S. Soskin, M.V. Vasnetsov, Manifestation of a hidden dislokation wave originated in a plane wave diffraction of on a half-plane // Int. Conf. Correlation Optics, O.V.Angelski, Editor, in Proc. SPIE, 3904, p. 18-25 (1999). 